\title{
One-dimensional diffusion in an asymmetric random environment
}

\author{
Dimitrios Cheliotis ${ }^{1}$ \\ Department of Mathematics, Bahen Center for Information Technology, 40 St. George St., 6th floor, Toronto, ON M5S 3G3, Canada
}

Received 11 October 2004; received in revised form 6 June 2005; accepted 30 August 2005

Available online 27 March 2006

\begin{abstract}
According to a theorem of S. Schumacher, for a diffusion $X$ in an environment determined by a stable process that belongs to an appropriate class and has index $a$, it holds that $X_{t} /(\log t)^{a}$ converges in distribution as $t \rightarrow \infty$ to a random variable having an explicit description in terms of the environment. We compute the density of this random variable in the case the stable process is spectrally one-sided. This computation extends a result of $\mathrm{H}$. Kesten and quantifies the bias that the asymmetry of the environment causes to the behavior of the diffusion.
\end{abstract}

(c) 2006 Elsevier Masson SAS. All rights reserved.

\section{Résumé}

Selon le théorème de $\mathrm{S}$. Schumacher, si l'on a une diffusion $X$ dans un environnement déterminé par un processus stable appartenant à une classe appropriée et avec un exposant $a$, il est établi que $X_{t} /(\log t)^{a}$ converge, à distribution que $t \rightarrow+\infty$, vers une variable aléatoire néanmoins spécifiquement identifiable selon les paramètres de l'environnement. Nous calculons alors la distribution de probabilité de la variable aléatoire dans le cas où le processus stable a un spectre unilatéral. Ce calcul prolonge la portée des résultats publiés par H. Kesten et quantifie l'influence que l'asymétrie de l'environnement a sur le comportement de la diffusion. (c) 2006 Elsevier Masson SAS. All rights reserved.

MSC: primary 60K37; secondary 60J60, 60G52

Keywords: Diffusion; Random environment; Renewal theorem; Stable process

\section{Introduction}

On the space $\mathcal{W}:=\left\{f \in \mathbb{R}^{\mathbb{R}}: f\right.$ is right continuous with left limits $\}$ consider the Skorohod topology, the $\sigma$-field of the Borel sets, and $\mathbb{P}$ a measure on $\mathcal{W}$.

Also let $\Omega:=C([0,+\infty))$, and equip it with the $\sigma$-field of Borel sets derived from the topology of uniform convergence on compact sets. For $w \in \mathcal{W}$, we denote by $\mathbf{P}_{w}$ the probability measure on $\Omega$ such that $\{\omega(t): t \geqslant 0\}$ is a diffusion with $\omega(0)=0$ and generator

$$
\frac{1}{2} \mathrm{e}^{w(x)} \frac{\mathrm{d}}{\mathrm{d} x}\left(\mathrm{e}^{-w(x)} \frac{\mathrm{d}}{\mathrm{d} x}\right)
$$

\footnotetext{
E-mail address: dimitris@ math.toronto.edu (D. Cheliotis).

URL: http://www.math.toronto.edu/dimitris/(D. Cheliotis).

1 Research partially supported by an anonymous Stanford Graduate Fellowship and by NSF grant DMS-0072331.
} 
The construction of such a diffusion is done with a scale and time transformation from a one-dimensional Brownian motion (see e.g. [5,16]). The diffusion does not explode in finite time if and only if $k_{w}(+\infty)=k_{w}(-\infty)=+\infty$, where $k_{w}$ is defined for all $x \in \mathbb{R}$ by $k_{w}(x):=\int_{0}^{x} \int_{0}^{y} \mathrm{e}^{w(y)-w(z)} \mathrm{d} y \mathrm{~d} z$. The last statement is Theorem 3 of [15]. We will only consider measures $\mathbb{P}$ on $\mathcal{W}$ with the property

$$
\mathbb{P}\left(k_{w}(+\infty)=k_{w}(-\infty)=+\infty\right)=1 .
$$

For $\mathbb{P}$-almost all $w \in \mathcal{W}, \omega$ satisfies the formal SDE

$$
\begin{aligned}
& \mathrm{d} \omega(t)=\mathrm{d} \beta(t)-\frac{1}{2} w^{\prime}(\omega(t)) \mathrm{d} t, \\
& \omega(0)=0,
\end{aligned}
$$

where $\beta$ is a one-dimensional standard Brownian motion.

Then consider the space $\mathcal{W} \times \Omega$, equip it with the product $\sigma$-field, and take the probability measure defined by

$$
\mathrm{d} \mathcal{P}(w, \omega)=\mathrm{d} \mathbf{P}_{w}(\omega) \mathrm{d} \mathbb{P}(w) .
$$

The marginal of $\mathcal{P}$ in $\Omega$ gives a process which is known as diffusion in random environment; the environment being the function $w$.

The following result, concerning a class of environments $\left(w_{t}\right)_{t \in \mathbb{R}}$, was proved by S. Schumacher $[13,14]$.

Fact 1. Assume (2) holds and that there is an a $>0$ such that the net of processes $\left\{\left(w(s t) / t^{1 / a}\right)_{s \in \mathbb{R}}: t>0\right\}$ converges to $\left(U_{s}\right)_{s \in \mathbb{R}}$ in the Skorohod topology in $\mathcal{W}$ as $t \rightarrow+\infty$, where $U$ satisfies

(i) $U$ is non-degenerate.

(ii) If $[x, y]$ is a finite interval, and if $\xi:=\inf \left\{s \geqslant x: U_{s}=\inf _{r \in[x, y]} U_{r}\right\}$, then $U$ is continuous at $\xi$.

(iii) If $[x, y]$ is a finite interval, $U$ attains each of the values $\inf _{r \in[x, y]} U_{r}, \sup _{r \in[x, y]} U_{r}$ only once in $[x, y]$.

(iv) $\mathbb{P}\left(\exists s>0\right.$ such that $\left.U_{s}>0\right)=\mathbb{P}\left(\exists s<0\right.$ such that $\left.U_{s}>0\right)=1$.

Then there is a process $b:[0, \infty) \times \mathcal{W} \rightarrow \mathbb{R}$ such that for the formal solution $\omega$ of (3) it holds

$$
\frac{\omega_{t}}{(\log t)^{a}}-b_{1}\left(w^{(\log t)}\right) \rightarrow 0 \quad \text { in } \mathcal{P} \text { as } t \rightarrow+\infty,
$$

where for $r>0$ we let $w^{(r)}(s)=r^{-1} w\left(s r^{a}\right)$ for all $s \in \mathbb{R}$.

This result shows the dominant effect of the environment, through the process $b$, on the asymptotic behavior of the diffusion.

In the case where $w$ is a two sided stable process with index $a \in(1,2]$ and having no positive jumps, we have $w^{(r)} \stackrel{\text { law }}{=} w$. Assuming that the assumptions of Fact 1 above are satisfied (we will prove this later), we get $\omega_{t} /(\log t)^{a} \Rightarrow$ $b_{1}(w)$ as $t \rightarrow+\infty$. The main result of this paper is the computation of the density of the random variable $b_{1}(w)$.

Let $E_{a}(z):=\sum_{n=0}^{\infty} z^{n} / \Gamma(1+a n)$. In the next section we will introduce two non-negative random variables with distribution functions $F_{u}, F_{d}$ respectively, having Laplace transforms

$$
\begin{aligned}
& \int_{0}^{\infty} \mathrm{e}^{-\lambda x} \mathrm{~d} F_{u}(x)=\left(E_{a}^{\prime}(\lambda)+\frac{a}{a-1} \lambda E_{a}^{\prime \prime}(\lambda)\right)^{-1}(\Gamma(a+1))^{-1}, \\
& \int_{0}^{\infty} \mathrm{e}^{-\lambda x} \mathrm{~d} F_{d}(x)=\Gamma(a+1)\left(E_{a}^{\prime}(\lambda)+\frac{a}{a-1} \lambda E_{a}^{\prime \prime}(\lambda)-\frac{a}{a-1} \lambda \frac{\left(E_{a}^{\prime}(\lambda)\right)^{2}}{E_{a}(\lambda)}\right),
\end{aligned}
$$

for all $\lambda>0$. And our main result is the following. 
Theorem. Let $w$ be a Lévy process with $\mathbb{E}\left(\mathrm{e}^{\lambda w_{t}}\right)=\mathrm{e}^{t \lambda^{a}}$ for all $\lambda, t \geqslant 0$, where a belongs to $(1,2]$. The density of $b_{1}(w)$ is

$$
f_{b_{1}}(x)= \begin{cases}(a-1) \Gamma(a)\left(1-F_{u}(-x)\right) & \text { for } x \leqslant 0, \\ (a-1) \Gamma(a)\left(1-F_{d}(x)\right) & \text { for } x>0 .\end{cases}
$$

Remark 1. When $a \neq 2$, the asymmetry of the environment has a visible effect on the path of the diffusion. We will show in Section 4 that $\mathbb{P}\left(b_{1}<0\right)=\gamma(a)$ for a strictly decreasing function $\gamma$ of $a$ in $[1,2]$ having $\gamma(1)=1$ and $\gamma(2)=1 / 2$. Since, by the theorem of Schumacher (Fact 1$), \lim _{t \rightarrow+\infty} \mathcal{P}\left(\omega_{t}<0\right)=\mathbb{P}\left(b_{1}<0\right)$, it follows that the diffusion is biased towards the left, and the bias increases as $a$ decreases to 1 . Of course, the diffusion is recurrent.

Remark 2. In the case $a=2$, the process $w / \sqrt{2}$ is a standard two sided Brownian motion and $E_{2}(z)=\cosh (\sqrt{z})$. The distribution functions $F_{u}, F_{d}$ coincide, and their Laplace transform, appearing above, equals $1 / \cosh (\sqrt{\lambda})$. Using Laplace inversion, we recover by the above theorem the well known result of Kesten [10].

The interest in the diffusions satisfying the assumptions of Fact 1 stems from the fact that they exhibit subdiffusive behavior; they are very slow. Compare the $(\log t)^{a}$ in Fact 1 with the $t^{1 / 2}$ for the analogous result for Brownian motion. First Sinai [17] studied the discrete time analog of diffusion in Brownian environment, which is random walk in random environment on $\mathbb{Z}$, and established the analogous to Fact 1 result with normalizing factor $(\log t)^{2}$. Shortly after, S. Schumacher studied the continuous time case.

When $w$ is a stable process having index $a$ and satisfying the assumptions of Fact $1, \omega_{t} /(\log t)^{a}$ converges in distribution to $b_{1}(w)$. The density of $b_{1}(w)$ has been computed under various assumptions for $w$. Kesten [10] considered the case where $w$ is a two sided Brownian motion. Golosov [8], the case where $w(s)=+\infty$ for $s<0$ and $(w(s))_{s} \geqslant 0$ a Brownian motion, i.e., there is a reflecting barrier at zero. Tanaka [18], the case where $w$ is a symmetric stable process. In the cases where $w$ equals $|B|$ or $-|B|$, with $B$ two sided Brownian motion having $B_{0}=0$, Schumacher's theorem does not apply (in the first case, (iii) fails; in the second, both (iii) and (iv) fail). However, Tanaka [19,18] showed that, in both cases, $\omega(t) /(\log t)^{2}$ converges in distribution to a symmetric random variable. For the restriction of each of the two variables on $[0,+\infty)$ he gave the Laplace transform. Ours is the first asymmetric case considered.

\section{Preliminaries}

\subsection{Definition of the process $b$}

For a function $w: \mathbb{R} \rightarrow \mathbb{R}, x>0$, and $y_{0} \in \mathbb{R}$, we say that $w$ admits an $x$-minimum at $y_{0}$ if there are $\alpha, \beta \in \mathbb{R}$ with $\alpha<y_{0}<\beta, w\left(y_{0}\right)=\inf \{w(y): y \in[\alpha, \beta]\}, w(\alpha) \geqslant w\left(y_{0}\right)+x$, and $w(\beta) \geqslant w\left(y_{0}\right)+x$. We say that $w$ admits an $x$-maximum at $y_{0}$ if $-w$ admits an $x$-minimum at $y_{0}$.

For convenience, we will call a point where $w$ admits an $x$-maximum or $x$-minimum, an $x$-maximum or an $x$-minimum respectively.

We denote by $R_{x}(w)$ the set of $x$-extrema of $w$ and define

$$
\mathcal{W}_{1}:=\left\{w \in \mathcal{W} \text { : for every } x>0 \text { the set } R_{x}(w) \text { has no accumulation point in } \mathbb{R},\right.
$$

it is unbounded above and below, and the points of $x$-maxima and $x$-minima alternate\}.

Thus, for $w \in \mathcal{W}_{1}$ and $x>0$, we can write $R_{x}(w)=\left\{x_{k}(w, x): k \in \mathbb{Z}\right\}$ with $\left(x_{k}(w, x)\right)_{k \in \mathbb{Z}}$ strictly increasing, $x_{0}(w, x) \leqslant 0<x_{1}(w, x), \lim _{k \rightarrow-\infty} x_{k}(w, x)=-\infty$, and $\lim _{k \rightarrow \infty} x_{k}(w, x)=\infty$. Whenever $\mathbb{P}\left(\mathcal{W}_{1}\right)=1$, which will be the case for us, the definition Schumacher gave for $b$ agrees with the following.

Definition 1. The process $b:[0,+\infty) \times \mathcal{W} \rightarrow \mathbb{R}$ is defined for $x>0$ and $w \in \mathcal{W}_{1}$ as

$$
b_{x}(w):= \begin{cases}x_{0}(w, x) & \text { if } x_{0}(w, x) \text { is an } x \text {-minimum, } \\ x_{1}(w, x) & \text { else }\end{cases}
$$

and $b_{x}(w)=0$ if $x=0$ or $w \in \mathcal{W} \backslash \mathcal{W}_{1}$. 


\subsection{Some useful facts}

In the case under consideration, the process $w$ appearing in (1) is a two sided stable process with index $a \in(1,2]$, no positive jumps, and $w(0)=0$. By two sided stable we mean that we take two i.i.d. stable processes $Y$, $\widetilde{Y}$ with paths in $D([0,+\infty)):=\left\{f \in \mathbb{R}^{[0,+\infty)}: f\right.$ right continuous with left limits $\}$, and define $w$ by $w_{s}=Y_{s}$ for $s \geqslant 0$ and $w_{s}=-\widetilde{Y}_{(-s)-}$ for $s<0$. Then $w$ has paths right continuous with left limits. Also it takes both positive and negative values. In fact for all $t \geqslant 0$, we have $\mathbb{P}\left(w_{t} \geqslant 0\right)=a^{-1}$ (see [2, VIII.1]).

Before stating two more properties of $w$, we remind the reader that for the given Lévy process, a point $x$ is called regular for a set $A \subset \mathbb{R}$ if $\mathbb{P}_{x}\left(\inf \left\{t>0: w_{t} \in A\right\}=0\right)=1$, where $\mathbb{P}_{x}$ is the law of the process starting at time 0 from $x$.

\section{Fact 2.}

(I) 0 is regular for $(0,+\infty)$ and $(-\infty, 0)$.

(II) $\underline{\lim }_{t \rightarrow \pm \infty} w_{t}=-\infty, \overline{\lim }_{t \rightarrow \pm \infty} w_{t}=+\infty$.

The first statement follows from Rogozin's criterion (Proposition VI.11 in [2]) and the fact that

$$
\int_{0}^{1} t^{-1} \mathbb{P}\left(w_{t} \geqslant 0\right) \mathrm{d} t=\int_{0}^{1} t^{-1} \mathbb{P}\left(w_{t} \leqslant 0\right) \mathrm{d} t=+\infty .
$$

The second statement follows from Theorem VI.12 in [2] and the fact that

$$
\int_{1}^{+\infty} t^{-1} \mathbb{P}\left(w_{t} \geqslant 0\right) \mathrm{d} t=\int_{1}^{+\infty} t^{-1} \mathbb{P}\left(w_{t} \leqslant 0\right) \mathrm{d} t=+\infty .
$$

The assumptions of Fact 1 are satisfied. Relation (2) holds because of Fact 2(II), and every process in the set $\left\{\left(w(s t) / t^{1 / a}\right)_{s \in \mathbb{R}}: t>0\right\}$ has the same law as $w$. So $U=w$. Then (ii) and (iii) follow from Lemma 3 (see Section 5), whose assumption holds (Fact 2(I) above), while (i) and (iv) are clearly true.

Also Fact 2 and Lemma 5 (see Section 5) show that $\mathbb{P}\left(\mathcal{W}_{1}\right)=1$, and so the process $b$ is determined by the definition of the previous subsection.

The absence of positive jumps implies that $\mathbb{E}\left(\exp \left\{\lambda w_{t}\right\}\right)<\infty$ for all $t, \lambda>0$ [2, VII.1]. Thus, the characteristic function of $w_{1}$ extends to an analytic function in $\{z \in \mathbb{C}: \operatorname{Im}(z) \leqslant 0\}$, and by its form [2, VIII.1] we can see that there is a positive constant $c$ such that

$$
\mathbb{E}\left(\exp \left\{\lambda w_{t}\right\}\right)=\exp \left\{c t \lambda^{a}\right\} \text { for all } t, \lambda>0 .
$$

In this work, we assume that $c=1$ as every other case reduces to this one after a normalization.

Remark 3. We stick to the spectrally negative case because for $w$ spectrally positive, the process $\tilde{w}$ defined by $\widetilde{w}_{t}=\lim _{s \nearrow-t} w_{s}$ for $t \in \mathbb{R}$ is spectrally negative stable with the same index and $b .(w)=-b .(\widetilde{w})$.

\section{Preparation and proof of the theorem}

If $w$ is under $\mathbb{P}$ a two sided stable process with $\mathbb{E}\left(\exp \left\{\lambda w_{t}\right\}\right)=\exp \left\{t \lambda^{a}\right\}$ for all $t, \lambda \geqslant 0$ and some $a \in(1,2]$, then $\mathbb{P}\left(\mathcal{W}_{1}\right)=1$ as was explained in the previous section. So for $x>0$ let $R_{x}(w)=\left\{x_{k}: k \in \mathbb{Z}\right\}$ be the set of $x$-extrema for $w$, with $\left(x_{k}\right)_{k \in \mathbb{Z}}$ strictly increasing and $x_{0} \leqslant 0<x_{1}$.

Lemma 1. The trajectories between consecutive $x$-extrema, $\left(w_{x_{k}+t}-w_{x_{k}}: t \in\left[0, x_{k+1}-x_{k}\right]\right)$ with $k \in \mathbb{Z}$, are independent, and the ones corresponding to even non-zero $k$ (respectively odd $k$ ) are identically distributed.

The proof of the lemma is given in Section 5. We call the translation $\left(w-w\left(x_{k}\right)\right) \mid\left[x_{k}, x_{k+1}\right]$ of the trajectory of $w$ between two consecutive $x$-extrema an $x$-slope (or a slope, when the value of $x$ is clear or irrelevant), a slope that takes only non-negative values an upward slope, and a slope taking only non-positive values a downward slope. We call $\left(w-w\left(x_{0}\right)\right) \mid\left[x_{0}, x_{1}\right]$ the central $x$-slope. 
Remark 4. As will become clear when we will examine the structure of the $k$-slopes in Section 4, the upward and downward $k$-slopes of a two sided Brownian motion (i.e., $a=2$ ), excluding the central one, are identically distributed up to sign change. When $a \neq 2$, since the process has only negative jumps, the upward $k$-slopes are essentially different from the downward.

For any $x$-slope $T:[\alpha, \beta] \rightarrow \mathbb{R}$ we call $l(T):=\beta-\alpha$ the length of $T$. Also we denote by $\theta(T)$ the slope with domain $[0, \beta-\alpha]$ and values $\theta(T)(\cdot)=T(\alpha+\cdot)$.

First we determine the distribution functions $F_{u}, F_{d}$ of the lengths $\underline{l}_{1}, \bar{l}_{1}$ of an upward and a downward 1-slope respectively from the common distributions mentioned in the preceding lemma. By scaling, this gives the laws for the $x$-slopes when $x \neq 1$. The proof of the following lemma is given in Section 4 .

Lemma 2. For all $u>0$,

$$
\begin{aligned}
& \mathbb{E}\left(\mathrm{e}^{-u \underline{l}_{1}}\right)=\left(\Gamma(a+1)\left(E_{a}^{\prime}(u)+\frac{a}{a-1} u E_{a}^{\prime \prime}(u)\right)\right)^{-1}, \\
& \mathbb{E}\left(\mathrm{e}^{-u \bar{l}_{1}}\right)=\Gamma(a+1)\left(E_{a}^{\prime}(u)+\frac{a}{a-1} u E_{a}^{\prime \prime}(u)-\frac{a}{a-1} u \frac{\left(E_{a}^{\prime}(u)\right)^{2}}{E_{a}(u)}\right) .
\end{aligned}
$$

In particular, the mean values of $\underline{l}_{1}, \bar{l}_{1}$ are

$$
\begin{aligned}
& \mathbb{E}\left(\underline{l}_{1}\right)=\frac{1}{a-1} \frac{\Gamma(a)}{\Gamma(2 a-1)}, \\
& \mathbb{E}\left(\bar{l}_{1}\right)=\frac{1}{a-1}\left(\frac{1}{\Gamma(a)}-\frac{\Gamma(a)}{\Gamma(2 a-1)}\right) .
\end{aligned}
$$

And now we are ready to prove our theorem.

Proof of the theorem. For $t \in \mathbb{R}$, let $T_{t}$ be the 1-slope around $t$. More precisely, $T_{t}$ is the slope with domain $\left(T_{t}\right)=$ $\left[c_{t}, d_{t}\right]$ and $t \in\left[c_{t}, d_{t}\right)$. And define

$$
q:=\inf \left\{t>0: T_{t} \text { downward slope with domain }\left(T_{t}\right) \subset(0,+\infty)\right\} .
$$

Then

$$
\mathbb{P}\left(b_{1}(w)>x\right)=\mathbb{P}\left(T_{0} \text { downward slope and } d_{0}>x\right)=\mathbb{P}\left(T_{t} \text { downward slope and } d_{t}-t>x\right)
$$

for all $t>0$ because $T_{0}(-t+\cdot)$ is the same as the slope around $t$ for $\left(w_{s-t}-w_{-t}: s \in \mathbb{R}\right)$, and the latter process has the same law as $w$. Call $C_{t}$ the event in the last probability. Then

$$
\mathbb{P}\left(C_{t}\right)=\mathbb{P}\left(C_{t} \text { and } q<t\right)+\mathbb{P}\left(C_{t} \text { and } q \geqslant t\right) .
$$

The second term goes to 0 as $t \rightarrow+\infty$. To work with the first term, we consider a sequence of independent random variables $\left(\xi_{n}\right)_{n} \geqslant 1$, with $\xi_{n}$ having distribution function $F_{d}$ if $n$ is odd, and $F_{u}$ if $n$ is even. Also let $\left(\zeta_{n}\right)_{n} \geqslant 1$ be a sequence of independent random variables with $\zeta_{n} \stackrel{\text { law }}{=} \xi_{n+1}$ for all $n \geqslant 1$.

Finally, define

$$
\begin{array}{ll}
S_{n}:=\xi_{1}+\cdots+\xi_{n}, & \widetilde{S}_{n}:=\zeta_{1}+\cdots+\zeta_{n}, \\
N(t):=\inf \left\{n \geqslant 1: S_{n} \geqslant t\right\}, & \widetilde{N}(t):=\inf \left\{n \geqslant 1: \widetilde{S}_{n} \geqslant t\right\}, \\
B_{t}:=S_{N(t)}-t, & \widetilde{B}_{t}:=\widetilde{S}_{\widetilde{N}(t)}-t .
\end{array}
$$

Then Lemma 1 implies that

$$
\mathbb{P}\left(C_{t} \text { and } q<t\right)=\int_{0}^{t} \mathbb{P}\left(N(t-y) \text { is odd and } B_{t-y}>x\right) \mathrm{d} F_{q}(y),
$$


where $F_{q}$ is the distribution function of $q$. We will show that the limit

$\lim _{t \rightarrow+\infty} \mathbb{P}\left(N(t)\right.$ is odd and $\left.B_{t}>x\right)$

exists, and we will compute its value.

Define $g_{1}, g_{2}:[0,+\infty) \rightarrow \mathbb{R}$ by

$$
\begin{aligned}
& g_{1}(t):=\mathbb{P}\left(N(t) \text { is odd and } B_{t}>x\right), \\
& g_{2}(t):=\mathbb{P}\left(\widetilde{N}(t) \text { is even and } \widetilde{B}_{t}>x\right)
\end{aligned}
$$

for all $t \geqslant 0$. Then $g_{1}(t)=\mathbb{P}\left(\xi_{1}>t+x\right)+\int_{0}^{t} g_{2}(t-s) \mathrm{d} F_{d}(s)$ and $g_{2}(t)=\int_{0}^{t} g_{1}(t-s) \mathrm{d} F_{u}(s)$. Consequently, $g_{1}(t)=\mathbb{P}\left(\xi_{1}>t+x\right)+g_{1} *\left(F_{u} * F_{d}\right)$, where $\left(F_{u} * F_{d}\right)(x):=\int_{\mathbb{R}} F_{u}(x-y) \mathrm{d} F_{d}(y)$ for all $x \in \mathbb{R}$ is the distribution function of $\xi_{1}+\xi_{2}$, and by the renewal theorem (see [6, Chapter 3, statement (4.9)])

$$
\lim _{t \rightarrow \infty} g_{1}(t)=\frac{1}{\mathbb{E}\left(\underline{l}_{1}\right)+\mathbb{E}\left(\bar{l}_{1}\right)} \int_{0}^{+\infty} \mathbb{P}\left(\xi_{1}>s+x\right) \mathrm{d} s=\frac{1}{\mathbb{E}\left(\underline{l}_{1}\right)+\mathbb{E}\left(\bar{l}_{1}\right)} \int_{x}^{+\infty} \mathbb{P}\left(\xi_{1}>s\right) \mathrm{d} s .
$$

By (5)-(7), this equals $\mathbb{P}\left(b_{1}(w)>x\right)$. Differentiating with respect to $x$ and noting that $\mathbb{E}\left(\underline{l}_{1}\right)+\mathbb{E}\left(\bar{l}_{1}\right)=((a-1)$, $\Gamma(a))^{-1}$ (by Lemma 2), we find the density of $b_{1}(w)$ in $[0,+\infty)$ as stated in the theorem. The density in $(-\infty, 0]$ is found similarly.

\section{Hitting times computations}

According to Lemma 1, excluding the central $k$-slope, the images of all upward (respectively downward) $k$-slopes under the map $\theta$ have the same distribution say $m_{k, u}$ (respectively $m_{k, d}$ ). In this section we describe the structure of a $k$-slope picked from either distribution.

Consider a Lévy process $\left(X_{t}\right)_{t \geqslant 0}$ starting from 0 and for which 0 is regular for $(-\infty, 0)$ and $(0,+\infty)$.

For $t>0$ define

$$
\begin{aligned}
& \underline{X}_{t}:=\inf \left\{X_{s}: s \in[0, t]\right\}, \\
& \bar{X}_{t}:=\sup \left\{X_{s}: s \in[0, t]\right\},
\end{aligned}
$$

and for $k>0$,

$$
\begin{aligned}
& \bar{\tau}_{k}:=\inf \left\{t>0: \bar{X}_{t}-X_{t} \geqslant k\right\}, \\
& \bar{\sigma}_{k}:=\sup \left\{s<\bar{\tau}_{k}: \bar{X}_{s}=X_{s}\right\}, \\
& \bar{\beta}_{k}:=\bar{X}_{\bar{\tau}_{k}}, \\
& \underline{\tau}_{k}:=\inf \left\{t>0: X_{t}-\underline{X}_{t} \geqslant k\right\}, \\
& \underline{\sigma}_{k}:=\sup \left\{s<\underline{\tau}_{k}: \underline{X}_{s}=X_{s}\right\}, \\
& \underline{\beta}_{k}:=-\underline{X}_{\underline{\tau}_{k}} .
\end{aligned}
$$

If $\bar{\tau}_{k}, \underline{\tau}_{k}<+\infty$ a.s., then $X$ is continuous at $\bar{\sigma}_{k}, \underline{\sigma}_{k}$, and splitting the path of $X$ at $\bar{\sigma}_{k}$ (or $\underline{\sigma}_{k}$ ) creates two independent pieces (see Lemmata 3 and 4 in Section 5).

In the following, we will use the operation of "gluing together" functions defined on compact intervals. For two functions $f:[\alpha, \beta] \rightarrow \mathbb{R}, g:[\gamma, \delta] \rightarrow \mathbb{R}$, by gluing $g$ to the right of $f$ we mean that we define a new function $j:[\alpha, \beta+$ $\delta-\gamma] \rightarrow \mathbb{R}$ with

$$
j(x)= \begin{cases}f(x) & \text { for } x \in[\alpha, \beta], \\ f(\beta)+g(x-\beta+\gamma)-g(\gamma) & \text { for } x \in[\beta, \beta+\delta-\gamma] .\end{cases}
$$

Clearly an upward $k$-slope picked from $m_{k, u}$ is obtained by gluing two independent trajectories with law $\left(X_{\underline{\sigma}_{k}+s}-\right.$ $\left.X_{\underline{\sigma}_{k}}: s \in\left[0, \underline{\tau}_{k}-\underline{\sigma}_{k}\right]\right),\left(X_{s}: s \in\left[0, \bar{\sigma}_{k}\right]\right)$ in this order, while a downward $k$-slope picked from $m_{k, d}$ is obtained by gluing two independent trajectories with law $\left(X_{\bar{\sigma}_{k}+s}-X_{\bar{\sigma}_{k}}: s \in\left[0, \bar{\tau}_{k}-\bar{\sigma}_{k}\right]\right),\left(X_{s}: s \in\left[0, \underline{\sigma}_{k}\right]\right)$ in this order. 
In the remaining of this section, we compute the Laplace transforms of the distributions of the lengths and heights of these four kinds of trajectories in the case that $X$ is a Lévy process with no positive jumps, and for which $\bar{\tau}_{k}, \underline{\tau}_{k}<+\infty$ a.s. In particular, we exclude the case where $X$ is the negative of a subordinator. As already mentioned in Section 2, the absence of positive jumps implies that $\mathbb{E}\left(\exp \left\{\lambda X_{t}\right\}\right)<\infty$ for all $\lambda>0$ (see [2, VII.1]). Let $\psi:[0,+\infty) \rightarrow \mathbb{R}$ be defined by

$$
\mathbb{E}\left(\exp \left\{\lambda X_{t}\right\}\right)=\exp \{t \psi(\lambda)\}
$$

for all $\lambda \geqslant 0$. It holds that $\psi$ is convex with $\psi(0)=0, \psi(+\infty)=+\infty$ (see [2, Chapter VII]). Denote by $\Phi(q)$ the largest root of $\psi(x)=q$. For every $q \geqslant 0$ there is a continuous function $W^{(q)}:[0,+\infty) \rightarrow[0,+\infty)$ with Laplace transform

$$
\int_{0}^{+\infty} \mathrm{e}^{-\lambda x} W^{(q)}(x) \mathrm{d} x=\frac{1}{\psi(\lambda)-q} \quad \text { for all } \lambda>\Phi(q) .
$$

The family of functions $\left\{W^{(q)}: q \geqslant 0\right\}$ appears in the solution of the exit problem for $X$. More specifically, if for $0<x<y$ we define $T:=\inf \left\{t>0: X_{t} \notin(0, y)\right\}$, then (see [4])

$$
\mathbb{E}_{x}\left(\mathrm{e}^{-q T} 1_{X_{T}=y}\right)=W^{(q)}(x) / W^{(q)}(y) \text { for all } q \geqslant 0 .
$$

For every $q \geqslant 0$, we define the function $Z^{(q)}:[0,+\infty) \rightarrow[1,+\infty)$ by

$$
Z^{(q)}(x)=1+q \int_{0}^{x} W^{(q)}(z) \mathrm{d} z .
$$

Note. In the following, instead of $W^{(0)}, Z^{(0)}$ we write just $W, Z$.

We also introduce a family of processes that is obtained from $X$ by a change of measure. More specifically, since for $c \geqslant 0$ the process $\left(\exp \left\{c X_{t}-\psi(c) t\right\}\right)_{t} \geqslant 0$ is a martingale with mean 1 , we can introduce the probability measure $\mathbb{P}^{c}$ for which

$$
\left.\frac{\mathrm{d} \mathbb{P}^{c}}{\mathrm{~d} \mathbb{P}}\right|_{\mathcal{F}_{t}}=\exp \left\{c X_{t}-\psi(c) t\right\} \quad \text { for all } t \geqslant 0 .
$$

It is easy to see that $X$ is under $\mathbb{P}^{c}$ a Lévy process with no positive jumps for which 0 is regular for $(-\infty, 0)$ and $(0,+\infty)$. Its Laplace exponent is given by $\psi_{c}(\lambda)=\psi(\lambda+c)-\psi(c)$ for $\lambda \geqslant 0$. We denote by $\mathbb{E}^{c}$ the expectation with respect to $\mathbb{P}^{c}$ and by $\Phi_{c}, W_{c}^{(q)}, Z_{c}^{(q)}$ the corresponding functions. As proved in [1], for fixed $c, x \geqslant 0$, the map $W_{c}^{(\cdot)}(x)$ can be extended uniquely to an analytic function in $\mathbb{C}$. The same holds for $Z_{c}^{(\cdot)}(x)$ obviously. A relation that we will use in the following is

$$
W_{c}^{(u-\psi(c))}(x)=\mathrm{e}^{-c x} W^{(u)}(x)
$$

for all $x, c \geqslant 0, u \in \mathbb{C}$. It is Remark 4 in [1].

The main result of this section is the following.

Proposition 1. Let $X$ be a spectrally negative Lévy process for which zero is regular for $(-\infty, 0)$ and $(0,+\infty)$, and $k>0$ such that $\bar{\tau}_{k}, \underline{\tau}_{k}<\infty$ a.s. Then for $u, v \geqslant 0$ it holds

$$
\begin{aligned}
& \mathbb{E}\left(\exp \left\{-u\left(\bar{\tau}_{k}-\bar{\sigma}_{k}\right)-v\left(\bar{X}_{\bar{\tau}_{k}}-X_{\bar{\tau}_{k}}-k\right)\right\}\right)=\mathrm{e}^{v k} \frac{W(k)}{W^{\prime}(k)}\left(Z_{v}^{(p)}(k) \frac{W_{v}^{(p) \prime}(k)}{W_{v}^{(p)}(k)}-p W_{v}^{(p)}(k)\right), \\
& \mathbb{E}\left(\exp \left\{-u \bar{\sigma}_{k}\right\} 1_{\bar{\beta}_{k} \leqslant x}\right)=\frac{W^{\prime}(k)}{W(k)} \frac{W^{(u)}(k)}{W^{(u) \prime}(k)}\left(1-\mathrm{e}^{-x W^{(u) \prime}(k) / W^{(u)}(k)}\right), \\
& \mathbb{E}\left(\exp \left\{-u\left(\underline{\tau}_{k}-\underline{\sigma}_{k}\right)\right\}\right)=\frac{W(k)}{W^{(u)}(k)},
\end{aligned}
$$




$$
\begin{aligned}
& \mathbb{E}\left(\exp \left\{-u \underline{\sigma}_{k}\right\}\right)=\frac{1}{Z^{(u)}(k)} \frac{W^{(u)}(k)}{W(k)}, \\
& \mathbb{E}\left(\exp \left\{-u \underline{\beta}_{k}\right\}\right)=\frac{\mathrm{e}^{-u k}}{Z_{u}^{(-\psi(u))}(k)},
\end{aligned}
$$

where $p=u-\psi(v)$.

Proof. Most of the formulas are contained in the computations in [1,11]. We provide the parts not treated there.

Relation (11) is relation (17) in p. 223 of [1].

Relation (12) is proved by modifying the argument in the computation of $I_{1}$ in pp. 222, 223 of the same paper. That is, we integrate up to local time $x$.

For the proof of relation (13) we will use facts and the standard notation from excursion theory (see e.g. [2, Chapter IV]). Denote by $D[0,+\infty)$ the space of real valued functions with domain $[0,+\infty)$ which are right continuous and have left limits everywhere. The set

$$
\mathcal{E}:=\{\varepsilon \in D[0,+\infty): \exists a \in(0,+\infty] \text { such that } \varepsilon(x) \neq 0 \text { for } x \in(0, a)\}
$$

is called the space of excursions. Together with $\mathcal{E}$, we introduce a new point $\delta$ whose use will appear shortly. Let $(L(t))_{t \geqslant 0}$ be a local time process at zero for $Y:=X-\underline{X}$, and define $L^{-1}(t):=\inf \{s \geqslant 0: L(s)>t\}$ for all $t>0$. The excursion process $\left(e_{t}\right)_{t>0}$ of $Y$ is given by

$$
e_{t}(s):= \begin{cases}Y_{L^{-1}(t-)+s} 1_{0 \leqslant s \leqslant L^{-1}(t)-L^{-1}(t-)} & \text { if } L^{-1}(t)-L^{-1}(t-)>0, \\ \delta & \text { otherwise, }\end{cases}
$$

for all $t>0, s \geqslant 0$. It is a Poisson point process with values in $\mathcal{E} \cup\{\delta\}$; we denote by $n$ its characteristic measure. For $\varepsilon \in \mathcal{E}$, we call $\bar{\varepsilon}:=\sup _{s \in[0, \zeta]} \varepsilon(s)$ the height of $\varepsilon$. Now returning to what we want to compute, observe that the random variable $\underline{\tau}_{k}-\underline{\sigma}_{k}$ is the time that it takes for the first excursion of $Y$ with height at least $k$ to reach $k$. The law of this excursion is $n(\varepsilon \mid \bar{\varepsilon} \geqslant k)$ (see [2, Chapter 0, Proposition 2]). Thus, the expectation we want is

$$
\int_{\mathcal{E}} \mathrm{e}^{-u \rho_{k}} \mathrm{~d} n(\varepsilon \mid \bar{\varepsilon} \geqslant k),
$$

where $\rho_{k}:=\inf \{t \geqslant 0: \varepsilon(t) \geqslant k\}$. For $\theta \in(0, k]$, we define $\rho_{\theta}:=\inf \{t \geqslant 0: \varepsilon(t) \geqslant \theta\}, \mathcal{G}_{\theta}:=\sigma\left(\varepsilon(t): t \leqslant \rho_{\theta}\right)$, and

$$
M_{\theta}:=\mathrm{e}^{-u \rho_{\theta}} \frac{W^{(u)}(\theta)}{W^{(u)}(k)} \frac{W(k)}{W(\theta)} .
$$

The denominator is not zero due to the assumption $\underline{\tau}_{k}<\infty$ a.s. and (9). We claim that $\left(M_{\theta}\right)_{\theta \in(0, k]}$ is a martingale with respect to the measure $n_{k}:=n(\cdot \mid \bar{\varepsilon} \geqslant k)$ and the filtration $\left(\mathcal{G}_{\theta}\right)_{\theta \in(0, k]}$. Denote by $\mathbb{E}_{\mu}$ the expectation with respect to any given measure $\mu$. Observe that $M_{k}=e^{-u \rho_{k}}$ and

$$
\mathbb{E}_{n_{k}}\left(M_{k} \mid \mathcal{G}_{\theta}\right)=\mathbb{E}_{n}\left(\mathrm{e}^{-u \rho_{k}} 1_{\rho_{k}<\infty} \mid \mathcal{G}_{\theta}\right) / \mathbb{E}_{n}\left(1_{\rho_{k}<\infty} \mid \mathcal{G}_{\theta}\right) .
$$

Using the Markov property for excursions (see [12, Theorem VI.48.1]), the absence of positive jumps, and (9), we see that the numerator equals

$$
\mathrm{e}^{-u \rho_{\theta}} \mathbb{E}_{\theta}\left(\mathrm{e}^{-u T_{k}^{+}} 1_{T_{k}^{+}<T_{0}^{-}}\right)=\mathrm{e}^{-u \rho_{\theta}} W^{(u)}(\theta) / W^{(u)}(k),
$$

where $\mathbb{E}_{\theta}$ is the expectation with respect to the law of $X$ starting from $\theta$, and

$$
\begin{aligned}
& T_{k}^{+}:=\inf \left\{t \geqslant 0: X_{t} \geqslant k\right\}, \\
& T_{0}^{-}:=\inf \left\{t \geqslant 0: X_{t} \leqslant 0\right\} .
\end{aligned}
$$

Now set $u=0$ in the last expression to find the value of the denominator in (16) as

$$
\mathbb{E}_{n}\left(1_{\rho_{k}<\infty} \mid \mathcal{G}_{\theta}\right)=W(\theta) / W(k) .
$$

Thus, $\mathbb{E}_{n_{k}}\left(M_{k} \mid \mathcal{G}_{\theta}\right)=M_{\theta}$ proving the claim. So

$$
\mathbb{E}_{n_{k}}\left(\mathrm{e}^{-u \rho_{k}}\right)=\mathbb{E}_{n_{k}}\left(M_{k}\right)=\mathbb{E}_{n_{k}}\left(M_{\theta}\right)=\frac{W^{(u)}(\theta)}{W^{(u)}(k)} \frac{W(k)}{W(\theta)} \mathbb{E}_{n_{k}}\left(\mathrm{e}^{-u \rho_{\theta}}\right)
$$


for all $\theta \in(0, k]$. Relation (13) will be proved if we show that the limit of the last quantity as $\theta \downarrow 0$ is $W(k) / W^{(u)}(k)$. Certainly $\lim _{\theta \downarrow 0} \mathbb{E}_{n_{k}}\left(\mathrm{e}^{-u \rho_{\theta}}\right)=1$ using the bounded convergence theorem. For the term $W^{(u)}(\theta) / W(\theta)$ we use relation (9) from [4], which is $W^{(u)}(\theta)=W(\theta)+\sum_{j=1}^{+\infty} u^{j} W^{*(j+1)}(\theta)$, and the bound $W^{*(j+1)}(\theta) \leqslant \theta^{j} W(\theta)^{j+1} / j$ ! (relation (10) in [4]). These give that $\lim _{\theta \downarrow 0} W^{(u)}(\theta) / W(\theta)=1$.

Relation (14) follows from (13), the independence of $\underline{\sigma}_{k}, \underline{\tau}_{k}-\underline{\sigma}_{k}$ (Lemma 4), and the expression for the Laplace transform of $\underline{\tau}_{k}$ given in [11, Proposition 2] as

$$
\mathbb{E}\left(\exp \left\{-u \underline{\tau}_{k}\right\}\right)=\frac{1}{Z^{(u)}(k)} \quad \text { for } u \geqslant 0 .
$$

Finally, for relation (15) we compute $\mathbb{E}\left(\exp \left\{-u \underline{\beta}_{k}\right\}\right)=\mathbb{E}\left(\exp \left\{u\left(k-\underline{\beta}_{k}\right)-u k\right\}\right)=\mathrm{e}^{-u k} \mathbb{E}\left(\exp \left\{u X_{\underline{\tau}_{k}}\right\}\right)$. For $n \in \mathbb{N}$ we have

$$
\begin{aligned}
\mathbb{E}\left(\exp \left\{u X_{\left(\underline{\tau}_{k} \wedge n\right)}\right\}\right) & =\mathbb{E}\left(\exp \left\{u X_{\left(\underline{\tau}_{k} \wedge n\right)}-\psi(u)\left(\underline{\tau}_{k} \wedge n\right)+\psi(u)\left(\underline{\tau}_{k} \wedge n\right)\right\}\right) \\
& =\mathbb{E}^{u}\left(\exp \left\{\psi(u)\left(\underline{\tau}_{k} \wedge n\right)\right\}\right) .
\end{aligned}
$$

Taking $n \rightarrow+\infty$ and applying the dominated convergence theorem in the first quantity (since $X_{\underline{\tau}_{k}} \leqslant k$ by the absence of positive jumps) and the monotone convergence theorem in the last quantity of the last relation, we obtain

$$
\mathbb{E}\left(\exp \left\{u X_{\underline{\tau}_{k}}\right\}\right)=\mathbb{E}^{u}\left(\exp \left\{\psi(u) \underline{\tau}_{k}\right\}\right) .
$$

To compute the last expectation, observe that relation (17) written for the spectrally negative Lévy process $\left(X, \mathbb{P}^{u}\right)$ is

$$
\mathbb{E}^{u}\left(\exp \left\{-v \underline{\tau}_{k}\right\}\right)=\frac{1}{Z_{u}^{(v)}(k)} \text { for } v \geqslant 0 .
$$

We will show that this holds for $v=-\psi(u)$ also. So assume that $\psi(u)>0$. The left hand side is finite for $v=-\psi(u)$ (due to (18) and $X_{\underline{\tau}_{k}} \leqslant k$ ), which implies that it can be written as a power series of $-v$ in $D(0, \psi(u)$ ) with positive coefficients, continuous in $\overline{D(0, \psi(u))}$. The denominator of the right-hand side can be extended to an entire function of $v$ as was mentioned just before this proposition. By a well known property of analytic functions, Eq. (19) will hold for all $v \in \overline{D(0, \psi(u))}$. Applying it for $v=-\psi(u)$ and combining it with the equalities before it, we obtain (15).

In the case of our interest, $\psi(u)=u^{a}$. Consequently,

$$
\begin{array}{ll}
\Phi(u)=u^{1 / a}, & W(z)=z^{a-1} / \Gamma(a), \\
W^{(u)}(z)=a z^{a-1} E_{a}^{\prime}\left(u z^{a}\right), & Z^{(u)}(k)=E_{a}\left(u k^{a}\right),
\end{array}
$$

for $z, u, v \in[0,+\infty)$. The expressions for $W, W^{(u)}$ are found in [3].

Proof of Lemma 2. Remember the description of $k$-slopes given in the beginning of this section just after the definition of the "gluing together" operation, where the role of $X$ is played now by $w$. It follows that the length of an upward 1-slope picked from $m_{1, u}$ equals in distribution to $\underline{\tau}_{1}-\underline{\sigma}_{1}+\bar{\sigma}_{1}^{*}$, where $\bar{\sigma}_{1}^{*}$ is independent of $\underline{\tau}_{1}-\underline{\sigma}_{1}$, and $\bar{\sigma}_{1}^{*} \stackrel{\text { law }}{=} \bar{\sigma}_{1}$. Similarly, the length of a downward 1-slope picked from $m_{1, d}$ equals in distribution to $\bar{\tau}_{1}-\bar{\sigma}_{1}+\underline{\sigma}_{1}^{*}$, with $\underline{\sigma}_{1}^{*}$ independent of $\bar{\tau}_{1}-\bar{\sigma}_{1}$, and $\underline{\sigma}_{1}^{*} \stackrel{\text { law }}{=} \underline{\sigma}_{1}$. Using relations (11)-(13), and (14), we get the formulas for the Laplace transforms. For the mean values of $\underline{l}_{1}, \bar{l}_{1}$, we compute the derivatives of their Laplace transforms at zero. To justify the move of the differentiation under the expectation, we use the monotone convergence theorem; which applies because the length of a slope is a positive random variable and the function $\left(\lambda \mapsto\left(1-\mathrm{e}^{-a \lambda}\right) / \lambda\right)$ is non-negative and decreasing in $\mathbb{R}$ for any $a>0$.

Justification of Remark 1. Using our theorem, the fact that $F_{u}$ is the distribution function for $\underline{l}_{1}$, and $\mathbb{E}\left(\underline{l}_{1}\right)+\mathbb{E}\left(\underline{l}_{1}\right)=$ $((a-1) \Gamma(a))^{-1}$, we obtain $\mathbb{P}\left(b_{1}<0\right)=\mathbb{E}\left(\underline{l}_{1}\right) /\left(\mathbb{E}\left(\underline{l}_{1}\right)+\mathbb{E}\left(\underline{l}_{1}\right)\right)=\mathrm{e}^{-g(a)}$, where

$$
g(a):=\log \left(\frac{\mathbb{E}\left(\bar{l}_{1}\right)}{\mathbb{E}\left(\underline{l}_{1}\right)}+1\right) .
$$


By Lemma 2,

$$
g(a)=\log \frac{\Gamma(2 a-1)}{\Gamma^{2}(a)},
$$

and using the formula (see $[7, \S 1.9$, relation (1)])

$$
\log \Gamma(z)=\int_{0}^{+\infty}\left[(z-1)-\frac{1-\mathrm{e}^{-(z-1) t}}{1-\mathrm{e}^{-t}}\right] \frac{\mathrm{e}^{-t}}{t} \mathrm{~d} t \quad \text { for } \operatorname{Re}(z)>0,
$$

we get

$$
g(a)=\int_{0}^{+\infty} \frac{\mathrm{e}^{-t}}{t\left(1-\mathrm{e}^{-t}\right)}\left(1-\mathrm{e}^{-(a-1) t}\right)^{2} \mathrm{~d} t .
$$

$g$ was defined above only for $a \in(1,2]$, but (20) extends it to a differentiable function in $(1 / 2,+\infty)$. It follows from (21) that $g$ is a strictly increasing function of $a$ in $[1,+\infty)$, and (20) shows that $g(1)=0, g(2)=\log 2$.

\section{Some lemmata}

In this section we prove some auxiliary results that we used above.

Proof of Lemma 1. Let $\bar{\tau}_{x,+}, \bar{\sigma}_{x,+}, \underline{\tau}_{x,+}, \underline{\sigma}_{x,+}$ be defined as in the beginning of Section 4 with $w$ having the role of $X$ and $k=x$. Similarly for $\bar{\tau}_{x,-}, \bar{\sigma}_{x,-}, \underline{\tau}_{x,-}, \underline{\sigma}_{x,-}$ with $\widehat{w}(s)=w(-s)$ for $s \geqslant 0$ having the role of $X$ and $k=x$. There are four possible cases for the ordering of the pairs $\left\{\bar{\tau}_{x,+}, \underline{\tau}_{x,+}\right\},\left\{\bar{\tau}_{x,-}, \underline{\tau}_{x,-}\right\}$. We treat only two of them, the other being similar.

First assume that $\bar{\tau}_{x,+}<\underline{\tau}_{x,+}$ and $\bar{\tau}_{x,-}<\underline{\tau}_{x,-}$. Then $\underline{\sigma}_{x,+}$ is a point of $x$-minimum for $w$, and the path of $w-w\left(\underline{\sigma}_{x,+}\right)$ after time $\underline{\sigma}_{x,+}$ is independent of the past by Lemma 4. Similarly, in the negative semi-axis, $-\underline{\sigma}_{x,-}$ is a point of $x$-minimum for $w$ and breaks the path of $w$ into two independent pieces. Between $-\underline{\sigma}_{x,-}, \underline{\sigma}_{x,+}$, there is exactly one more $x$-extremum. It is an $x$-maximum and it is the point in $\left\{-\bar{\sigma}_{x,-}, \bar{\sigma}_{x,+}\right\}$ where $w$ has greater value. Say it is $-\bar{\sigma}_{x,-}$. Then in the notation of the lemma, we have $x_{-1}=-\underline{\sigma}_{x,-}, x_{0}=-\bar{\sigma}_{x,-}$, and $x_{1}=\underline{\sigma}_{x,+}$. The points $x_{-1}, x_{0}, x_{1}$ cut the path of $w$ into four independent pieces. This, combined with the fact that $\left(w_{s}\right)_{s \leqslant 0} \stackrel{\text { law }}{=}$ $\left(-w_{(-s)-}\right)_{s \leqslant 0}$ and time reversal [2, Lemma II.2], shows that all upward $x$-slopes, including $w-w\left(x_{-1}\right) \mid\left[x_{-1}, x_{0}\right]$, are obtained by gluing two trajectories with law $\left(w_{s+\underline{\sigma}_{x,+}}-w_{\underline{\sigma}_{x,+}}: s \in\left[0, \underline{\tau}_{x,+}-\underline{\sigma}_{x,+}\right]\right),\left(w_{s}: s \in\left[0, \bar{\sigma}_{x,+}\right]\right)$ in this order, while all downward $x$-slopes, excluding $w-w\left(x_{0}\right) \mid\left[x_{0}, x_{-1}\right]$, are obtained by gluing two trajectories with law $\left(w_{s+\bar{\sigma}_{x,+}}-w_{\bar{\sigma}_{x,+}}: s \in\left[0, \bar{\tau}_{x,+}-\bar{\sigma}_{x,+}\right]\right),\left(w_{s}: s \in\left[0, \underline{\sigma}_{x,+}\right]\right)$. This description accounts for all $x$-slopes in the path decomposition of $w$.

Now assume that $\bar{\tau}_{x,+}<\underline{\tau}_{x,+}$ and $\bar{\tau}_{x,-}>\underline{\tau}_{x,-}$. Then $-\bar{\sigma}_{x,-}$ is a point of $x$-maximum for $w$, and $\underline{\sigma}_{x,+}$ is a point of $x$-minimum for $w$. If $w\left(\bar{\sigma}_{x,+}\right)-w\left(-\underline{\sigma}_{x,-}\right)<x$, then $x_{0}=-\bar{\sigma}_{x,-}, x_{1}=\underline{\sigma}_{x,+}$. If $w\left(\bar{\sigma}_{x,+}\right)-w\left(-\underline{\sigma}_{x,-}\right) \geqslant x$, then $x_{0}=-\underline{\sigma}_{x,-}, x_{1}=\bar{\sigma}_{x,+}$. As in the previous case, we get the desired description for the decomposition of the path of $w$ into $x$-slopes.

For a function $f:[0,+\infty) \rightarrow \mathbb{R}$ with only jump discontinuities and $x_{0}>0$, we say that $f$ has a left local maximum (respectively minimum) at $x_{0}$ if there is an $\varepsilon>0$ such that $f(x) \leqslant f\left(x_{0}-\right)$ (respectively $f(x) \geqslant f\left(x_{0}-\right)$ ) for all $x \in\left(x_{0}-\varepsilon, x_{0}\right)$. Similarly for a right local maximum and minimum.

Lemma 3. Let $X$ be a Lévy process such that 0 is regular for $(0,+\infty)$ and $(-\infty, 0)$. With probability one

(i) $X$ is continuous at every one sided local extremum.

(ii) In no two local minima (respectively maxima) $X$ has the same value.

Proof. (i) It is enough to consider the case of a local one sided maximum (the case of one sided local minimum follows by applying the present case to the process $-X$ ). Consider the set of times where the process jumps upwards 
or downwards by at least $1 / n$; it is a countable subset of $(0,+\infty)$ with no accumulation point. Apply the strong Markov property to each of these times. Since 0 is regular for $(0,+\infty)$, none of these can be a point of a right local maximum. Using time reversal [2, Lemma II.2] and the fact that 0 is regular for $(-\infty, 0)$, we exclude the existence of left local maxima.

(ii) This holds for any Lévy process that is not compound Poisson [2, Proposition VI.4]. It is a simple application of the strong Markov property.

In the next lemma, we use the notation introduced in the beginning of Section 4.

Lemma 4. Let $\left(X_{t}\right)_{t \geqslant 0}$ be a Lévy process starting from zero such that 0 is regular for $(-\infty, 0)$ and $(0,+\infty)$, and $\underline{\lim }_{t \rightarrow \infty} X_{t}=-\infty, \varlimsup_{\lim _{t \rightarrow \infty}} X_{t}=+\infty$. With probability one:

(i) The two trajectories $\left(X_{t}: t \in\left[0, \bar{\sigma}_{k}\right]\right)$ and $\left(X_{\bar{\sigma}_{k}}-X_{\bar{\sigma}_{k}+t}: t \in\left[0, \bar{\tau}_{k}-\bar{\sigma}_{k}\right]\right)$ are independent.

(ii) The two trajectories $\left(X_{t}: t \in\left[0, \underline{\sigma}_{k}\right]\right)$ and $\left(X_{\underline{\sigma}_{k}}-X_{\underline{\sigma}_{k}+t}: t \in\left[0, \underline{\tau}_{k}-\underline{\sigma}_{k}\right]\right)$ are independent.

This follows from the discussion in [9, Section 4]. So we do not give its proof. For the next statement, recall that the set $\mathcal{W}_{1}$ was defined in Section 1.

Lemma 5. If $\left(w_{t}\right)_{t \in \mathbb{R}}$ is a RCLL version of a Lévy process starting from zero such that 0 is regular for $(-\infty, 0)$ and $(0,+\infty), \underline{\lim }_{t \rightarrow \pm \infty} w_{t}=-\infty$, and $\overline{\lim }_{t \rightarrow \pm \infty} w_{t}=+\infty$, then $\mathbb{P}\left(\mathcal{W}_{1}\right)=1$

Proof. First we prove that for fixed $x>0$, the set

$$
C_{x}:=\left\{w \in C(\mathbb{R}): R_{x}(w) \text { has the properties appearing in the definition of } \mathcal{W}_{1}\right\}
$$

has $\mathbb{P}\left(C_{x}\right)=1$. To see this, observe that for $z$ a point of $x$-minimum and $\alpha_{z}=\sup \{\alpha<z: w(\alpha) \geqslant w(z)+x\}, \beta_{z}=$ $\inf \{\beta>z: w(\beta) \geqslant w(z)+x\}$ it holds that $\alpha_{z}<z<\beta_{z}$ (because $w$ is continuous at $z$ by Lemma 3(i)) and there is no other $x$-minimum in $\left(\alpha_{z}, \beta_{z}\right)$. Indeed, if $\tilde{z}$ is an $x$-minimum in $\left(\alpha_{z}, z\right)$, then in case $\beta_{\tilde{z}}>z$ we get that $w$ takes the same value in two local minima, while in case $\beta_{\tilde{z}}<z$ we get $w\left(\beta_{\tilde{z}}\right) \geqslant w(z)+x$. The first case is excluded by Lemma 3(ii), and the second contradicts the definition of $\alpha_{z}$. If $\tilde{z}$ is an $x$-minimum in $\left(z, \beta_{z}\right)$, then in case $\alpha_{z}<z$ we get that $w$ takes the same value in two local minima, while in case $\alpha_{\tilde{z}}>z$ we get $w\left(\alpha_{\tilde{z}}-\right) \geqslant w(z)+x$. The first case is excluded by Lemma 3(ii), and the second contradicts the definition of $\beta_{z}$.

Assume that there is a strictly monotone, say increasing, sequence $\left(z_{n}\right)_{n \geqslant 1}$ of $x$-minima converging to $z_{\infty} \in \mathbb{R}$. Then by the above observation we get $\overline{\lim }_{y, \tilde{y} \nearrow_{\infty}}(w(y)-w(\tilde{y})) \geqslant x$ implying that $w$ cannot have left limit at $z_{\infty}$. A contradiction with the fact that $w$ is RCLL. Similarly if $\left(z_{n}\right)_{n \geqslant 1}$ is decreasing. So in a set of $w$ 's in $\mathcal{W}$ with probability 1 , it holds that the set of $x$-minima of $w$ has no accumulation point. The same holds for the set of $x$-maxima, and as a result also for $R_{x}(w)$. Since $\underline{\lim }_{|t| \rightarrow \infty} w_{t}=-\infty$ and $\overline{\lim }_{|t| \rightarrow \infty} w_{t}=+\infty$, it follows that $\mathbb{P}\left(R_{x}(w)\right.$ is unbounded above and below $)=1$. Now between two consecutive $x$-maxima (respectively minima) there is exactly one $x$-minimum (respectively $x$-maximum). Indeed, take $z_{1}<z_{2}$ two consecutive $x$-minima, and call $s_{0}$ the unique point where $w$ attains its maximum in $\left[z_{1}, z_{2}\right]$. Then $w\left(s_{0}\right) \geqslant \max \left\{w\left(x_{1}\right), w\left(z_{2}\right)\right\}+x$. Because if $w\left(s_{0}\right)<w\left(x_{1}\right)+x$, then $\beta_{z_{1}}>z_{2}$, while if $w\left(s_{0}\right)<w\left(z_{2}\right)+x$, then $\alpha_{z_{2}}<z_{1}$, and both $\beta_{z_{1}}>z_{2}, \alpha_{z_{2}}<z_{1}$ are false as was shown above. So $s_{0}$ is an $x$-maximum. There is no other $\mathrm{x}$-maximum in $\left[z_{1}, z_{2}\right]$ because then we would find an $x$-minimum in $\left(z_{1}, z_{2}\right)$, which cannot happen since $z_{1}, z_{2}$ are consecutive $x$-minima. Consequently, $\mathbb{P}\left(C_{x}\right)=1$.

Finally, note that for all $n \in \mathbb{N} \backslash\{0\}$ we have $R_{n}(w) \subset R_{x}(w) \subset R_{1 / n}(w)$ for $x \in[1 / n, n]$, from which it follows that $\mathcal{W}_{1}=\bigcap_{x \in(0,+\infty)} C_{x}=\bigcap_{n \in \mathbb{N} \backslash\{0\}}\left(C_{n} \cap C_{1 / n}\right)$. Thus, $\mathbb{P}\left(\mathcal{W}_{1}\right)=1$.

\section{Acknowledgements}

My advisor, Amir Dembo, and the anonymous referee pointed out to me some errors in previous drafts of this paper. I am grateful to both of them. I also thank the referee for comments that greatly improved the structure and exposition of the paper. 


\section{References}

[1] F. Avram, A. Kyprianou, M. Pistorius, Exit problems for spectrally negative Lévy processes and applications to (Canadized) Russian options, Ann. Appl. Probab. 14 (1) (2004) 215-238.

[2] J. Bertoin, Lévy Processes, Cambridge University Press, Cambridge, 1996.

[3] J. Bertoin, On the first exit time of a completely asymmetric stable process from a finite interval, Bull. London Math. Soc. 28 (5) (1996) $514-520$.

[4] J. Bertoin, Exponential decay and ergodicity of completely asymmetric Lévy processes in a finite interval, Ann. Appl. Probab. 7 (1997) 156-169.

[5] T. Brox, A one-dimensional diffusion process in a Wiener medium, Ann. Probab. 14 (4) (1986) 1206-1218.

[6] R. Durrett, Probability: Theory and Examples, second ed., Wadsworth Pub. Co., 1996.

[7] A. Erdélyi, W. Magnus, F. Oberhettinger, F. Tricomi, Higher Transcendental Functions, vol. 1, McGraw-Hill Book Company, Inc., New York, 1953. Based, in part, on notes left by Harry Bateman.

[8] A.O. Golosov, Limit distributions for random walks in random environments, Soviet Math. Dokl. 28 (1983) 18-22.

[9] P. Greenwood, J. Pitman, Fluctuation identities for Lévy processes and splitting at the maximum, Adv. Appl. Probab. 12 (1980) 893-902.

[10] H. Kesten, The limit distribution of Sinai's random walk in random environment, Phys. A 138 (1-2) (1986) $299-309$.

[11] M. Pistorius, On exit and ergodicity of the spectrally one-sided Lévy process reflected at its infimum, J. Theoret. Probab. 17 (1) (2004) $183-220$.

[12] L.C.G. Rogers, D. Williams, Diffusions, Markov Processes, and Martingales, vol. 2, John Wiley and Sons, Inc., New York, 1987.

[13] S. Schumacher, Diffusions with random coefficients, in: Particle Systems, Random Media and Large Deviations, in: Contemp. Math., vol. 41, Amer. Math. Soc., Providence, RI, 1985.

[14] S. Schumacher, Diffusions with Random Coefficients, Ph.D. thesis, UCLA, 1984.

[15] P. Seignourel, Discrete schemes for processes in random media, Probab. Theory Related Fields 118 (3) (2000) $293-322$.

[16] Z. Shi, Sinai's walk via stochastic calculus, in: F. Comets, E. Pardoux (Eds.), Milieux Aléatoires, in: Panoramas et Synthèses, vol. 12, Société Mathématique de France, 2001.

[17] Y. Sinai, The limiting behavior of a one-dimensional random walk in a random medium, Theory Probab. Appl. 27 (1982) $256-268$.

[18] H. Tanaka, Limit distributions for one-dimensional diffusion processes in self-similar random environments, in: Hydrodynamic Behavior and Interacting Particle Systems, in: IMA Vol. Math. Appl., vol. 9, Springer, 1987, pp. 189-210.

[19] H. Tanaka, Limit distribution for 1-dimensional diffusion in a reflected Brownian medium, in: Séminaire de Probabilités, XXI, in: Lecture Notes in Math., vol. 1247, Springer, 1987, pp. 246-261. 